\title{
Oka's conjecture on irreducible plane sextics. II
}

Article in Journal of Knot Theory and Its Ramifications · February 2007

DOI: $10.1142 /$ S0218216509007348 · Source: arXiv

CITATIONS

17

1 author:

Alex Degtyarev

Bilkent University

81 PUBLICATIONS 748 CITATIONS

SEE PROFILE
READS

22

Some of the authors of this publication are also working on these related projects:

Project $\quad$ Lines in $\$$ K3\$-surfaces View project

Project $\quad$ Projective spaces in Fermat varieties View project 


\title{
OKA'S CONJECTURE ON IRREDUCIBLE PLANE SEXTICS
}

\author{
Alex Degtyarev
}

\begin{abstract}
We partially prove and partially disprove Oka's conjecture on the fundamental group/Alexander polynomial of an irreducible plane sextic. Among other results, we enumerate all irreducible sextics with simple singularities admitting dihedral coverings and find examples of Alexander equivalent Zariski pairs of irreducible sextics.
\end{abstract}

\section{INTRODUCTION}

1.1. Motivation and principal results. In 23, O. Zariski initiated the study of the fundamental group of the complement of a plane curve as a topological tool controlling multiple planes ramified at the curve. He found an example of a curve whose group is not abelian: it is a sextic with six ordinary cusps which all lie on a conic. Since then, very few general results have been obtained in this direction; one may mention M. V. Nori's theorem [15, stating that a curve with sufficiently simple singularities has abelian fundamental group, and two generalizations of original Zariski's example, due to B. G. Moishezon [13] and M. Oka [16].

The fundamental group of an algebraic curve $C$ of large degree is extremely difficult to compute. As an intermediate tool, Zariski 24] suggested to study its Alexander polynomial $\Delta_{C}(t)$, which proved quite useful in knot theory. This approach was later developed by A. Libgober in [11, 12. The Alexander polynomial is an algebraic invariant of a group; it is trivial whenever the group is abelian (see Section 3.1 for definitions and further references). In the case of plane curves, the Alexander polynomial can be found in terms of dimensions of certain linear systems, which depend on the types of the singular points of the curve and on their global position in $\mathbb{P}^{2}$, see 4 . As a disadvantage, the Alexander polynomial is often trivial, as it is subject to rather strong divisibility conditions, see [24, [11, and [6]. For example, it is trivial for all irreducible curves of degree up to five.

The fundamental groups of all curves of degree up to five, both irreducible and reducible, are known, see [5], and next degree six has naturally become a subject of intensive research. A number of contributions has been made by E. Artal, J. Carmona, J. I. Cogolludo, C. Eyral, M. Oka, H. Tokunaga, etc., see recent survey [18. As a result, it was discovered that an important rôle is played by the so called sextics of torus type, i.e., those whose equation can be represented in the form $p^{3}+q^{2}=0$, where $p$ and $q$ are some homogeneous polynomials of degree 2 and 3 , respectively.

2000 Mathematics Subject Classification. Primary: 14H30; Secondary: 14J28.

Key words and phrases. Plane sextic, torus type, K3-surface, Zariski pair, Alexander polynomial, dihedral covering, fundamental group. 
Among sextics of torus type is Zariski's six cuspidal sextic, as well as all other irreducible sextics with abnormally large Alexander polynomial, see [4]. Furthermore, sextics of torus type are a principal source of examples of irreducible curves with nontrivial Alexander polynomial or nonabelian fundamental group. Based on the known examples, Oka suggested the following conjecture.

1.1.1. Conjecture (Oka, see [10]). Let $C$ be an irreducible plane sextic, which is not of torus type. Then:

(1) the Alexander polynomial $\Delta_{C}(t)$ is trivial;

(2) if all singularities of $C$ are simple, the group $\pi_{1}\left(\mathbb{P}^{2} \backslash C\right)$ is abelian;

(3) the fundamental group $\pi_{1}\left(\mathbb{P}^{2} \backslash C\right)$ is abelian.

In this paper, we disprove parts (2) and (3) of the conjecture and prove part (1) restricted to sextics with simple singularities (i.e., those of type $\mathbf{A}_{p}, \mathbf{D}_{q}, \mathbf{E}_{6}, \mathbf{E}_{7}$, or $\mathbf{E}_{8}$, see [1] or [9] for their definition).

1.1.2. Theorem (see Theorem 4.1 .1 for details). An irreducible plane sextic $C$ with simple singularities is of torus type if and only if $\Delta_{C}(t) \neq 1$.

1.1.3. Theorem (see Theorems 4.3 .4 and 4.3 .3 for details). There are irreducible plane sextics $C_{1}, C_{2}$ with simple singularities whose fundamental groups factor to the dihedral groups $\mathbb{D}_{10}$ and $\mathbb{D}_{14}$, respectively. The sextics are not of torus type.

1.1.4. Theorem (see Theorem 5.2 .2 for details). There is an irreducible plane sextic $C$ with a singular point adjacent to $\mathbf{X}_{9}$ (a quadruple point) and fundamental group $\mathbb{D}_{10} \times(\mathbb{Z} / 3 \mathbb{Z})$. The sextic is not of torus type.

Theorems 1.1.2 1.1.4 are mere simplified versions of the statements cited in the titles. We do not prove them separately.

Essentially, Theorem 1.1.2 follows from the Riemann-Roch theorem for $K 3$ surfaces, which is not applicable if the curve has non-simple singular points. For sextics with a singular point adjacent to $\mathbf{X}_{9}$, we prove an analog of Theorem 1.1.2 (see Theorem 5.2.2) by calculating the fundamental groups directly. This result substantiates Conjecture 1.1.1(1) in its full version. The remaining case of curves with a singular point adjacent to $\mathbf{J}_{10}$ (a quasihomogeneous singularity of type $(3,6)$ ) requires a different approach; I am planning to treat it in a subsequent paper.

1.2. Other results. The bulk of the paper is related to the study of irreducible sextics with simple singularities whose fundamental groups factor to a dihedral group $\mathbb{D}_{2 n}, n \geqslant 3$. We call such curves special. Alternatively, special is an irreducible sextic that serves as the ramification locus of a regular $\mathbb{D}_{2 n}$-covering of the plane. We show that only $\mathbb{D}_{6}, \mathbb{D}_{10}$, and $\mathbb{D}_{14}$ can appear as monodromy groups of dihedral coverings ramified at irreducible sextics, see Theorem 4.3.2, and essentially enumerate all special sextics, see Sections 4.1 and 4.3 . (The list of sets of singularities realized by irreducible sextics with exactly one $\mathbb{D}_{6}$-covering is omitted due to its length, and the rigid isotopy classification of sextics admitting $\mathbb{D}_{6}$-coverings is not completed.)

As a by-product, we discover six sets of singularities that are realized by both special and non-special irreducible sextics with $\Delta_{C}(t)=1$. They give rise to so called Alexander equivalent Zariski pairs of irreducible sextics (see Remark 4.3.5 for details and further references). To my knowledge, these examples are new. It is worth mentioning that, as in the case of abundant vs. non-abundant curves (Zariski 
pairs of irreducible sextics that differ by their Alexander polynomials, see [4]), within each pair the special curve is distinguished by the existence of certain conics passing in a prescribed way through its singular points. One may hope that, as in the case of abundant curves, these conics can be used to obtain explicit equations.

The fundamental groups of special sextics are not known. I would suggest that, at least for the simplest curve in each set, they are minimal.

1.2.1. Conjecture. The fundamental groups of the special sextics with the sets of singularities $3 \mathbf{A}_{6}$ and $4 \mathbf{A}_{4}$ are $\mathbb{D}_{14} \times(\mathbb{Z} / 3 \mathbb{Z})$ and $\mathbb{D}_{10} \times(\mathbb{Z} / 3 \mathbb{Z})$, respectively.

Any reduced sextic $C$ of torus type is the critical locus of the projection to $\mathbb{P}^{2}$ of an irreducible cubic surface $V \subset \mathbb{P}^{3}$. The monodromy of this (irregular) covering is an epimorphism from $\pi_{1}\left(\mathbb{P}^{2} \backslash C\right)$ to the symmetric group $\mathbb{S}_{3}=\mathbb{D}_{6}$. Conversely, any such epimorphism gives rise to a triple covering of $\mathbb{P}^{2}$ ramified at $C$. We show that the existence of a torus structure is equivalent to the existence of an epimorphism $\pi_{1}\left(\mathbb{P}^{2} \backslash C\right) \rightarrow \mathbb{S}_{3}$, see Theorem 4.1.1. (The relation between $\mathbb{S}_{3}$-coverings and torus structures was independently discovered by Tokunaga [21.) Remarkably, it is not true that every triple plane obtained in this way is a cubic surface. In the world of irreducible sextics with simple singularities, there is one counter-example; it is given by Theorem 4.1 .3 .

The relation between torus structures and $\mathbb{D}_{6}$-coverings is exploited to detect sextics of torus type and eventually prove Theorem 1.1.2, Among other results, we classify irreducible sextics admitting more than one torus structure. The maximal number is attained at the famous nine cuspidal sextic: it has twelve torus structures and thirteen $\mathbb{D}_{6}$-coverings.

Our study of dihedral coverings is based on Proposition 3.4.4, which relates the existence of such coverings to a certain invariant $\mathcal{K}_{C}$ used in the classification of sextics. As a first step towards reducible curves, we prove Theorem 3.5.1, which takes into account the 2-torsion of the group. Still, this approach can only detect dihedral quotients of the fundamental group that are compatible with the standard homomorphism $\pi_{1}\left(\mathbb{P}^{2} \backslash C\right) \rightarrow \mathbb{Z} / 2 \mathbb{Z}$ sending each van Kampen generator to 1 . A somewhat complementary approach was developed by Tokunaga, see recent paper 20] for further references. In particular, he constructed a series of dihedral coverings of the plane ramified at reducible sextics. In the examples of [20, components of the ramification locus have distinct ramification indices.

Apart from the common goal, Conjecture 1.1.1, last section [5] is not related to the rest of the paper: it is a straightforward application of the results of [3] and [5] dealing with curves of degree $m$ with a singular point of multiplicity $m-2$. In Theorem 5.2.1, we enumerate all irreducible sextics with a quadruple point and nonabelian fundamental group. There are seven rigid isotopy classes; five of them are of torus type, and the remaining two have trivial Alexander polynomial.

1.3. Contents of the paper. In $₫ 2$, we introduce basic notation and remind a few facts needed in the sequel. $\$ 3$ contains a few auxiliary results, both old and new, related to sextics, Alexander polynomials, and torus structures. We introduce the notion of weight of a curve, which is used in subsequent statements. Proposition 3.4 .4 and Theorem 3.5 .1 are also proved here. In $\S \S 4$ and 5 , we state and prove extended versions of Theorems 1.1.2 1.1.4. The most involved is the case of curves admitting $\mathbb{D}_{6}$-coverings. Technical results obtained in Section 4.2 can be used in a further study of reducible sextics of torus type. 


\section{Preliminaries}

2.1. Basic notation. For an abelian group $G$, we use the notation $G^{*}$ for the dual group $\operatorname{Hom}(G, \mathbb{Z})$. The minimal number of generators of $G$ is denoted by $\ell(G)$; if $p$ is a prime, we abbreviate $\ell_{p}(G)=\ell\left(G \otimes \mathbb{F}_{p}\right)$.

We use the notation $\mathbb{B}_{n}$ for the braid group on $n$ strings, $\mathbb{S}_{n}$ for the symmetric group of degree $n$, and $\mathbb{D}_{2 n}$ for the dihedral group of order $2 n$, i.e., the semidirect product

$$
1 \rightarrow(\mathbb{Z} / n \mathbb{Z})[t] /(t+1) \rightarrow \mathbb{D}_{2 n} \rightarrow \mathbb{Z} / 2 \mathbb{Z} \rightarrow 1
$$

One has $\mathbb{S}_{3}=\mathbb{D}_{6}$. The reduced braid group is the quotient $\mathbb{B}_{n} / \Delta^{2}$ of $\mathbb{B}_{n}$ by its center. $\mathbb{B}_{3} / \Delta^{2}$ is the free product $(\mathbb{Z} / 2 \mathbb{Z}) *(\mathbb{Z} / 3 \mathbb{Z})$.

The Milnor number of an isolated singular point $P$ is denoted by $\mu(P)$. The Milnor number $\mu(C)$ of a reduced plane curve $C$ is defined as the total Milnor number of all singular points of $C$. Given two plane curves $C$ and $D$ and an intersection point $P \in C \cap D$, we use the notation $(C \cdot D)_{P}$ for the local intersection index of $C$ and $D$ at $P$.

When a statement is not followed by a proof, either because it is obvious or because it is cited from another source, it is marked with

2.2. Lattices. A lattice is a finitely generated free abelian group $L$ equipped with a symmetric bilinear form $b: L \otimes L \rightarrow \mathbb{Z}$. Usually, we abbreviate $b(x, y)=x \cdot y$ and $b(x, x)=x^{2}$. A lattice $L$ is even if $x^{2}=0 \bmod 2$ for all $x \in L$. As the transition matrix between two integral bases has determinant \pm 1 , the determinant $\operatorname{det} L=\operatorname{det} b \in \mathbb{Z}$ is well defined. A lattice $L$ is called nondegenerate if $\operatorname{det} L \neq 0$; it is called unimodular if $\operatorname{det} L= \pm 1$.

The bilinear form on a lattice $L$ extends to $L \otimes \mathbb{Q}$. If $L$ is nondegenerate, the dual group $L^{*}$ can be identified with the subgroup

$$
\{x \in L \otimes \mathbb{Q} \mid x \cdot y \in \mathbb{Z} \text { for all } x \in L\} .
$$

Hence, $L$ is a subgroup of $L^{*}$ and the quotient $L^{*} / L$ is a finite group; it is called the discriminant group of $L$ and is denoted by discr $L$. The discriminant group inherits from $L \otimes \mathbb{Q}$ a symmetric bilinear form discr $L \otimes \operatorname{discr} L \rightarrow \mathbb{Q} / \mathbb{Z}$, called the discriminant form, and, if $L$ is even, its quadratic extension $\operatorname{discr} L \rightarrow \mathbb{Q} / 2 \mathbb{Z}$. When speaking about discriminant groups, their (anti-)isomorphisms, etc., we assume that the discriminant form and its quadratic extension are taken into account. One has $|\operatorname{discr} L|=|\operatorname{det} L|$; in particular, $\operatorname{discr} L=0$ if and only if $L$ is unimodular.

Given a lattice $L$, we use the notation $n L, n \in \mathbb{N}$, for the orthogonal sum of $n$ copies of $L$, and $L(q), q \in \mathbb{Q}$, for the lattice obtained from $L$ by multiplying the bilinear form by $q$ (assuming that the result is an integral lattice).

From now on, all lattices are assumed even.

A root in a lattice $L$ is a vector of square $(-2)$. A root system is a negative definite lattice generated by its roots. Each root system admits a unique decomposition into orthogonal sum of irreducible root systems, the latter being either $\mathbf{A}_{p}, p \geqslant 1$, or $\mathbf{D}_{q}$, $q \geqslant 4$, or $\mathbf{E}_{6}, \mathbf{E}_{7}, \mathbf{E}_{8}$. Their discriminant forms are as follows:

$$
\begin{gathered}
\operatorname{discr} \mathbf{A}_{p}=\left\langle-\frac{p}{p+1}\right\rangle, \quad \operatorname{discr} \mathbf{D}_{2 k+1}=\left\langle-\frac{2 k+1}{4}\right\rangle, \quad \operatorname{discr} \mathbf{D}_{2 k}=\left[\begin{array}{cc}
-\frac{k}{2} & \frac{1}{2} \\
\frac{1}{2} & 1
\end{array}\right], \\
\operatorname{discr} \mathbf{E}_{6}=\left\langle\frac{2}{3}\right\rangle, \quad \operatorname{discr} \mathbf{E}_{7}=\left\langle\frac{1}{2}\right\rangle, \quad \operatorname{discr} \mathbf{E}_{8}=0 .
\end{gathered}
$$


Here, $\langle p / q\rangle$ with $(p, q)=1$ and $p q=0 \bmod 2$ represents the quadratic form on the cyclic group $\mathbb{Z} / q \mathbb{Z}$ sending 1 to $(p / q) \bmod 2 \mathbb{Z}$, and the $(2 \times 2)$-matrix represents a quadratic form on the group $(\mathbb{Z} / 2 \mathbb{Z})^{2}$.

A finite index extension of a nondegenerate lattice $L$ is a lattice $S$ containing $L$ as a finite index subgroup, so that the bilinear form on $L$ is the restriction of that on $S$. Since $S$ is a lattice, it is canonically embedded into $L^{*}$ and the quotient $\mathcal{K}=S / L$ is a subgroup of $\operatorname{discr} L$. This subgroup is called the kernel of the extension $S \supset L$. It is isotropic, i.e., the restriction to $\mathcal{K}$ of the discriminant quadratic form is identically zero. Conversely, given an isotropic subgroup $\mathcal{K} \subset \operatorname{discr} L$, the group $S=\left\{u \in L^{*} \mid(u \bmod L) \in \mathcal{K}\right\}$ is a finite index extension of $L$.

2.2.1. Theorem (see [14]). Let $L$ be a nondegenerate even lattice. Then the map $S \mapsto \mathcal{K}=S / L \subset \operatorname{discr} L$ establishes a one to one correspondence between the set of isomorphism classes of finite index extensions $S \supset L$ and the set of isotropic subgroups of $\operatorname{discr} L$. Under this correspondence, one has $\operatorname{discr} S=\mathcal{K}^{\perp} / \mathcal{K}$.

2.3. Singularities. Let $f(x, y)$ be a germ at an isolated singular point $P$, let $\tilde{X}$ be the minimal resolution of the singular point $P$ of the surface $z^{2}+f(x, y)=0$, and let $E_{i}$ be the irreducible components of the exceptional divisor in $\tilde{X}$. The group $H_{2}(\tilde{X})$ is spanned by the classes $e_{i}=\left[E_{i}\right]$, which are linearly independent and form a negative definite lattice with respect to the intersection index form. This lattice is called the resolution lattice of $P$ and is denoted $\Sigma(P)$. The basis $\left\{e_{i}\right\}$ is called a standard basis of $\Sigma(P)$; it is defined up to reordering. As usual, $e_{i}^{*}$ stand for the elements of the dual basis of $\Sigma(P)^{*}=H^{2}(\tilde{X})$.

If $P$ is simple, of type $\mathbf{A}, \mathbf{D}, \mathbf{E}$, then $\Sigma(P)$ is the irreducible root system of the same name and one has $\mu(P)=\operatorname{rk} \Sigma(P)$. In this case, we order the elements of a standard basis according to the following diagrams:

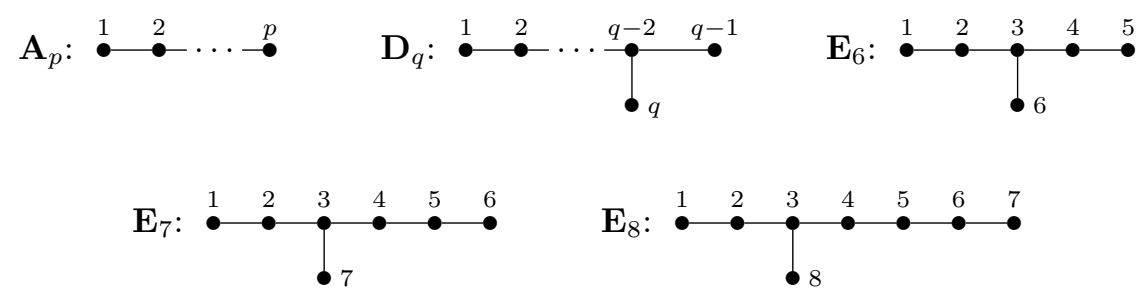

The order is still defined up to symmetries of the Dynkin graph.

A rigid isotopy of plane curves is a topologically equisingular deformation or, equivalently, a path in a topologically equisingular stratum of the space of curves. If all singular points involved are simple, the choice of the category (topological) in the definition above is irrelevant, as topologically equivalent simple singularities are diffeomorphic (see, e.g., [1] or [9]).

\section{Plane sextics}

3.1. Ramified coverings. Let $C \subset \mathbb{P}^{2}$ be a reduced plane sextic. Throughout 
the paper we use the notation introduced in the following diagram:

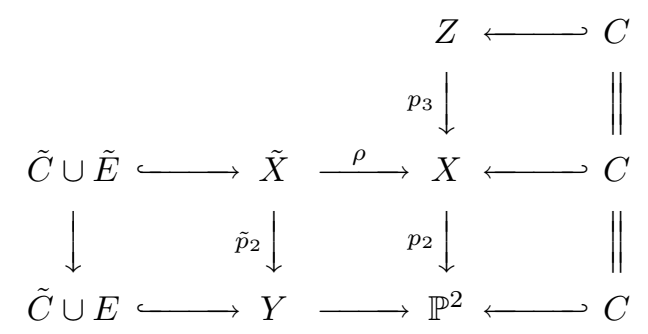

Here, $X$ and $Z$ are, respectively, the double and the 6 -fold cyclic coverings of $\mathbb{P}^{2}$ ramified at $C$; clearly, $Z$ can also be regarded as a triple covering of $X$. The copies of $C$ in $X$ and $Z$ are identified with $C$ itself. The map $\rho: \tilde{X} \rightarrow X$ is the minimal resolution of singularities of $X, \tilde{C} \subset \tilde{X}$ is the proper transform of $C$, and $\tilde{E}$ is the exceptional divisor. The restriction $\rho: \tilde{X} \backslash(\tilde{C} \cup \tilde{E})=X \backslash C$ is a diffeomorphism. If all singularities of $C$ are simple, then $\tilde{X}$ can be obtained as a double covering of a certain embedded resolution $Y$ of $C$; more precisely, $Y$ is the minimal resolution in which all odd order components of the pull-back of $C$ are smooth and disjoint. The exceptional divisor in $Y$ is denoted by $E$.

Let $C_{1}, \ldots, C_{r}$ be the irreducible components of $C$, and let $\operatorname{deg} C_{i}=m_{i}$. From the Poincaré duality it follows that the abelinization of $\pi_{1}\left(\mathbb{P}^{2} \backslash C\right)$ is the group $H_{1}\left(\mathbb{P}^{2} \backslash C\right)=\left(\mathbb{Z} c_{1} \oplus \ldots \oplus \mathbb{Z} c_{r}\right) / \sum m_{i} c_{i}$, where $c_{i}$ is the generator of $H^{2}\left(C_{i}\right)$ corresponding to the complex orientation of $C_{i}$. The map $c_{i} \mapsto 1, i=1, \ldots, r$, defines canonical epimorphisms $\pi_{1}\left(\mathbb{P}^{2} \backslash C\right) \rightarrow \mathbb{Z} / 6 \mathbb{Z} \rightarrow \mathbb{Z} / 2 \mathbb{Z}$. We consider their kernels

$$
\begin{aligned}
& K_{2}(C)=\operatorname{Ker}\left[\pi_{1}\left(\mathbb{P}^{2} \backslash C\right) \rightarrow \mathbb{Z} / 2 \mathbb{Z}\right]=\pi_{1}(X \backslash C), \\
& K_{6}(C)=\operatorname{Ker}\left[\pi_{1}\left(\mathbb{P}^{2} \backslash C\right) \rightarrow \mathbb{Z} / 6 \mathbb{Z}\right]=\pi_{1}(Z \backslash C)
\end{aligned}
$$

and their abelinizations

$$
\bar{K}_{2}(C)=H_{1}(X \backslash C), \quad \bar{K}_{6}(C)=H_{1}(Z \backslash C),
$$

respectively. The deck translations of the coverings $p_{2}$ and $p_{2} \circ p_{3}$ induce certain automorphisms $\operatorname{tr}_{2}$ of $\bar{K}_{2}(C)$ and $\operatorname{tr}_{6}$ of $\bar{K}_{6}(C)$, respectively; the deck translation of $p_{3}$ induces $\operatorname{tr}_{6}^{2}$ on $\bar{K}_{6}(C)$. Group theoretically, $\operatorname{tr}_{2}$ and $\operatorname{tr}_{6}$ are induced by the conjugation by the generators of $\mathbb{Z} / 2 \mathbb{Z}$ and $\mathbb{Z} / 6 \mathbb{Z}$, respectively.

The Alexander polynomial $\Delta_{C}(t)$ of a reduced sextic $C$ can be defined as the characteristic polynomial of the deck translation automorphism $\operatorname{tr}_{6}$ of the $\mathbb{C}$-vector space $\bar{K}_{6}(C) \otimes \mathbb{C}=H_{1}(Z \backslash C ; \mathbb{C})$. The definition in terms of $\bar{K}_{6}$ applies to any group $G$ equipped with a distinguished epimorphism $G \rightarrow \mathbb{Z} / 6 \mathbb{Z}$. One always has $\Delta_{C}(t) \mid(t-1)\left(t^{6}-1\right)^{4}$, see [11, and $\Delta_{C}(t)$ is defined over $\mathbb{Q}$; hence, it is a product of cyclotomic polynomials. If $C$ is irreducible, then $\Delta_{C}(t) \mid\left(t^{2}-t+1\right)^{3}$, see [4]. Alternative definitions of the Alexander polynomial of a plane curve and its basic properties can be found in the original paper [11] or recent survey [18. For the particular case of sextics, see [4] or [17].

3.1.1. Proposition. If $C$ is an irreducible plane sextic with $\Delta_{C}(t) \neq 1$, then the fundamental group $\pi_{1}\left(\mathbb{P}^{2} \backslash C\right)$ factors to the symmetric group $\mathbb{S}_{3}$. 
Proof. Since $\Delta_{C}(t) \neq 1$, the 3 -group $\operatorname{Hom}\left(\bar{K}_{6}(C), \mathbb{F}_{3}\right)$ is nontrivial and its order 3 automorphism $\operatorname{tr}_{6}^{2}$ has a fixed element. Hence, $\pi_{1}\left(\mathbb{P}^{2} \backslash C\right)$ has a quotient $G$ which is included into the exact sequence

$$
1 \rightarrow \mathbb{Z} / 3 \mathbb{Z} \rightarrow G \rightarrow \mathbb{Z} / 6 \mathbb{Z} \rightarrow 1
$$

so that $\operatorname{tr}_{6}^{2}$ acts identically on the kernel. (Here, $\operatorname{tr}_{6}$ is regarded as a generator of the quotient $\mathbb{Z} / 6 \mathbb{Z}$.) Since the abelinization of $\pi_{1}\left(\mathbb{P}^{2} \backslash C\right)$ is $\mathbb{Z} / 6 \mathbb{Z}$, the extension cannot be central. Hence, $\operatorname{tr}_{6}$ acts on the kernel via - id, the exact sequence splits, and $G$ factors to $\mathbb{D}_{6}=\mathbb{S}_{3}$.

3.2. Sextics of torus type. A reduced plane sextic $C$ is said to be of torus type if its equation can be represented in the form

$$
p^{3}\left(x_{0}, x_{1}, x_{2}\right)+q^{2}\left(x_{0}, x_{1}, x_{2}\right)=0
$$

where $p$ and $q$ are some homogeneous polynomials of degree 2 and 3 , respectively. A sextic is of torus type if and only if it is the critical locus of a projection to $\mathbb{P}^{2}$ of a cubic surface $V \subset \mathbb{P}^{3}$; the latter is given by $3 x_{3}^{3}+3 x_{3} p+2 q=0$. If $C$ is reduced, then $V$ has isolated singularities and, hence, is irreducible.

A representation (3.2.1), considered up to scalar multiples, is called a torus structure of $C$. Each torus structure gives rise to a conic $Q=\{p=0\}$ and a cubic $K=\{q=0\}$. With few exceptions, each conic $Q$ is obtained in this way from at most one torus structure. (In the exceptional cases, either $C$ contains $2 Q$ as a non-reduced component or $C$ consists of six lines through a single point.)

Each intersection point $P \in Q \cap K$ is a singular point for $C$; such points are called inner singularities of $C$ (with respect to the given torus structures). The other singular points that $C$ may have are called outer. A simple calculation using normal forms at $P$ shows that an inner singular point can be of type

- $\mathbf{A}_{3 k-1}$, if $K$ is nonsingular at $P$ and $(Q \cdot K)_{P}=k$, or

- $\mathbf{E}_{6}$, if $K$ is singular at $P$ and $(Q \cdot K)_{P}=2$, or

- adjacent to $\mathbf{J}_{10}$ (in the notation of [1]) otherwise.

Informally, the inner singularities and their types are due to the topology of the mutual position of $Q$ and $K$, whereas outer singularities occur accidentally in the family $(\alpha p)^{3}+(\beta q)^{2}=0$ under some special values of parameters $\alpha, \beta \in \mathbb{C}$. A sextic of torus type is called tame if all its singularities are inner. The rigid isotopy classification of irreducible tame sextics is found in [4].

3.2.2. Remark. In the case of non-simple points, one should probably speak about 'outer degenerations' of inner singularities. For example, if $P$ is a node for $K$ and $(Q \cdot K)_{P}=3$, the generic inner singularity at $P$ is of type $\mathbf{J}_{10}=\mathbf{J}_{2,0}$. However, under an appropriate choice of the parameters, it may degenerate to $\mathbf{J}_{2,1}$ or $\mathbf{J}_{2,2}$. This fact makes the study of sextics of torus type with non-simple singularities more involved.

3.2.3. Proposition. Let $C$ be a reduced sextic of torus type. Then the group $\pi_{1}\left(\mathbb{P}^{2} \backslash C\right)$ factors to the reduced braid group $\mathbb{B}_{3} / \Delta^{2}$ and to the symmetric group $\mathbb{S}_{3}$, and the Alexander polynomial $\Delta_{C}(t)$ has at least one factor $t^{2}-t+1$.

Proof. All statements follow immediately from the fact that any sextic of torus type can be perturbed to Zariski's six cuspidal sextic $C^{\prime}$, which is obtained from $Q$ and $K$ intersecting transversally at six points. Hence, there is an epimorphism $\pi_{1}\left(\mathbb{P}^{2} \backslash C\right) \rightarrow \pi_{1}\left(\mathbb{P}^{2} \backslash C^{\prime}\right)$. As shown in $[23], \pi_{1}\left(\mathbb{P}^{2} \backslash C^{\prime}\right)=\mathbb{B}_{3} / \Delta^{2}$. 
3.2.4. Definition. Let $P$ be a simple singular point, and let $\Sigma=\Sigma(P)$ be its resolution lattice. Define the weight $w(P)$ as follows:

$$
w(P)=\left\{\begin{array}{l}
\min \left\{-\frac{3}{2} u^{2} \mid u \in \Sigma^{*} \backslash \Sigma, 3 u \in \Sigma\right\}, \\
0, \quad \text { if }(\operatorname{discr} \Sigma) \otimes \mathbb{F}_{3}=0 .
\end{array}\right.
$$

The weight of a curve $C$ is the sum of the weights of its singular points.

3.2.5. Lemma. One has $w\left(\mathbf{A}_{3 k-1}\right)=k, w\left(\mathbf{E}_{6}\right)=2$, and $w(P)=0$ otherwise. In a standard basis $\left\{e_{i}\right\}$ of $\Sigma(P)$, the minimal value of $-\frac{3}{2} u^{2}$ as in Definition 3.2 .4 is attained, among other vectors, at $e_{k}^{*}$ or $e_{2 k}^{*}$ for $\mathbf{A}_{3 k-1}$ and at $e_{2}^{*}$ or $e_{4}^{*}$ for $\mathbf{E}_{6}$.

Proof. Since discr $\mathbf{E}_{6}=\left\langle\frac{2}{3}\right\rangle$, the integer $(3 u)^{2}$ must be $6 \bmod 18$. The maximal negative integer with this property is $-12=\left(3 e_{2}^{*}\right)^{2}=\left(3 e_{4}^{*}\right)^{2}$.

Let $\Sigma=\mathbf{A}_{3 k-1}$. Consider the standard representation of $\Sigma$ as the orthogonal complement of the characteristic element $\sum v_{i} \in \bigoplus \mathbb{Z} v_{i}, v_{i}^{2}=-1,1 \leqslant i \leqslant 3 k$. An element $u$ as in the definition of weight has the form $\frac{1}{3} \sum m_{i} v_{i}$ with $m_{i}=1 \bmod 3$ and $\sum m_{i}=0$. From the relation $(m-3)^{2}+(n+3)^{2}=\left(m^{2}+n^{2}\right)-6[(m-n)-3]$ it follows that, whenever two coefficients in the representation of $3 u$ differ more than by 3 , the value of $(3 u)^{2}$ can be increased. Hence, the coefficients of a square maximizing vector $3 u$ take only two values, which must be $2 k$ copies of 1 and $k$ copies of $(-2)$. Thus, the maximal square is $(3 u)^{2}=-6 k$.

For any other irreducible root system $\Sigma$ one has $(\operatorname{discr} \Sigma) \otimes \mathbb{F}_{3}=0$.

3.2.6. Remark. From comparing the values given by Lemma 3.2 .5 and those found in [4] it follows that, whenever $w(P) \neq 0$, one has $w(P)=d_{5 / 6}(P)$, where

$$
d_{5 / 6}(P)=\#\{s \in \operatorname{Spec}(P) \mid s \leqslant-1 / 6\}
$$

are the numbers introduced in 4 in conjunction with the Alexander polynomial. (See [1] for the definition of spectrum.) Roughly, $d_{5 / 6}(P)$ is the number of conditions imposed by $P$ on the linear system $\mathcal{L}_{5}$ of conics evaluating $\Delta_{C}(t)$.

Comparing Lemma 3.2 .5 and the list of inner singularities above, one concludes that, for a curve $C$ of torus type and conic $Q=\{p=0\}$ defined by a torus structure, $(Q \cdot C)_{P}=2 w(P)$ at each simple inner singular point $P$. (In particular, if all inner points are simple, then $w(C) \geqslant \sum_{P \in Q} w(P)=6$.) The following theorem, which we restate in terms of weights, asserts that this property is characteristic for conics arising from torus structures.

3.2.7. Theorem (see [4] or [19]). Let $C$ be a reduced sextic, and let $Q$ be a conic (not necessarily irreducible or reduced) intersecting $C$ at simple singular points so that, at each intersection point $P$, one has $(Q \cdot C)_{P}=w(P)$. Then $C$ has a torus structure (3.2.1) such that $Q$ is the conic $\{p=0\}$.

The statement proved in [4] is stronger than Theorem 3.2.7 it suffices to require that the inequality $(Q \cdot C)_{P} \geqslant d_{5 / 6}(P)$ hold at each intersection point $P$. In particular, the intersection points are not restricted a priori to $\mathbf{A}_{3 k-1}$ or $\mathbf{E}_{6}$.

3.3. The case of simple singularities. Let $C$ be a plane sextic with simple singularities only. Then all singular points of $X$ are also simple, and $\tilde{X}$ is a $K 3$ surface. Introduce the following notation:

- $L_{C}=H_{2}(\tilde{X})$ is the intersection lattice of $\tilde{X}$; 
- $h \in L_{C}$ is the class of the pull-back of a generic line in $\mathbb{P}^{2}$; one has $h^{2}=2$;

- $\Sigma_{C} \subset L_{C}$ is the sublattice spanned by the exceptional divisors;

- $S_{C}=\Sigma_{C} \oplus \mathbb{Z} h$;

- $\tilde{\Sigma}_{C}$ and $\tilde{S}_{C}$ are the primitive hulls of, respectively, $\Sigma_{C}$ and $S_{C}$ in $L_{C}$;

- $\mathcal{K}_{C} \subset \operatorname{discr} S_{C}$ is the kernel of the finite index extension $\tilde{S}_{C} \supset S_{C}$.

As is known, $L_{C}$ is the only unimodular even lattice of signature $(3,19)$ (one can take $L_{C} \cong 2 \mathbf{E}_{8} \oplus 3 \mathbf{U}$, where $\mathbf{U}$ is the hyperbolic plane), and $\Sigma_{C}$ is the orthogonal sum of the resolution lattices of all singular points of $C$.

When a curve $C$ is understood, we omit subscript $C$ in the notation.

The deck translation of the covering $p_{2}: X \rightarrow \mathbb{P}^{2}$ lifts to $\tilde{X}$ and permutes the components of $\tilde{E}$; hence, $\operatorname{tr}_{2}$ induces a certain automorphism of the Dynkin graph of $\Sigma_{C}$. The following lemma is an easy exercise using the embedded resolution $Y$ described in Section 3.1.

3.3.1. Lemma. For each simple singular point $P$ of $C$, the automorphism induced by $\operatorname{tr}_{2}$ on the Dynkin graph $D$ of $\Sigma(P)$ is the only nontrivial symmetry of $D$, if $P$ is of type $\mathbf{A}_{p}$ or $\mathbf{D}_{2 k+1}$, and the identity otherwise. As a consequence, the induced automorphism of discr $\Sigma(P)$ is the multiplication by $(-1)$.

The root system $\Sigma_{C}$ is called the set of singularities of $C$. (Since $\Sigma_{C}$ admits a unique decomposition into irreducible summands, it does encode the number and the types of the singular points.) The triple $h \in S_{C} \subset L_{C}$ is called the homological type of $C$. It is equipped with a natural orientation $\theta_{C}$ of maximal positive definite subspaces in $S_{C}^{\perp} \otimes \mathbb{R}$; it is given by the real and imaginary parts of the class realized in $H^{2}(\tilde{X} ; \mathbb{C})=L_{C} \otimes \mathbb{C}$ by a holomorphic 2 -form on $\tilde{X}$.

3.3.2. Definition. An (abstract) set of (simple) singularities is a root system. A configuration extending a set of singularities $\Sigma$ is a finite index extension $\tilde{S} \supset S=$ $\Sigma \oplus \mathbb{Z} h, h^{2}=2$, satisfying the following conditions:

(1) the primitive hull $\tilde{\Sigma}=h_{\tilde{S}}^{\perp}$ of $\Sigma$ in $\tilde{S}$ has no roots other than those in $\Sigma$;

(2) there is no root $r \in \Sigma$ such that $\frac{1}{2}(r+h) \in \tilde{S}$.

3.3.3. Definition. An abstract homological type extending a set of singularities $\Sigma$ is an extension of $S=\Sigma \oplus \mathbb{Z} h, h^{2}=2$, to a lattice $L \cong 2 \mathbf{E}_{8} \oplus 3 \mathbf{U}$ such that the primitive hull $\tilde{S}$ of $S$ in $L$ is a configuration extending $\Sigma$. An abstract homological type is encoded by the triple $h \in S \subset L$, so that $\mathbb{Z} h$ is a direct summand in $S$ and $h_{S}^{\perp}=\Sigma$. An isomorphism of two abstract homological types $h^{\prime} \in S^{\prime} \subset L^{\prime}$ and $h^{\prime \prime} \in S^{\prime \prime} \subset L^{\prime \prime}$ is an isometry $L^{\prime} \rightarrow L^{\prime \prime}$ taking $h^{\prime}$ to $h^{\prime \prime}$ and $S^{\prime}$ onto $S^{\prime \prime}$. An orientation of an abstract homological type $h \in S \subset L$ is an orientation $\theta$ of maximal positive definite subspaces in $S_{L}^{\perp} \otimes \mathbb{R}$.

3.3.4. Theorem (see [7). The homological type $h \in S_{C} \subset L_{C}$ of a plane sextic $C$ with simple singularities is an abstract homological type; two sextics are rigidly isotopic if and only if their oriented homological types are isomorphic. Conversely, any oriented abstract homological type is isomorphic to the oriented homological type of a plane sextic with simple singularities.

The existence part of Theorem 3.3.4 was first proved by J.-G. Yang [22].

3.3.5. Remark. The principal steps of the classification of abstract homological types are outlined in [7]. A configuration $\tilde{S} \supset S$ is determined by its kernel $\mathcal{K}$, 
which plays an important rôle in the sequel. The existence of a primitive extension $\tilde{S} \subset L$ reduces to the existence of an even lattice $N$ of signature $(2,20-\operatorname{rk} \tilde{S})$ and discriminant $-\operatorname{discr} \tilde{S}$; it can be detected using Theorem 1.10.1 in [14]. Finally, for the uniqueness one needs to know that

(1) a lattice $N$ as above is unique up to isomorphism,

(2) each automorphism of $\operatorname{discr} N=-\operatorname{discr} \tilde{S}$ is induced by an isometry of either $N$ or $\Sigma$, and

(3) the homological type has an orientation reversing automorphism.

In most cases considered in this paper, (1) and (2) can be derived from, respectively, Theorems 1.13.2 and 1.14.2 in [14], and (3) follows from the existence of a vector of square 2 in $N$. (The case when $N$ is a definite lattice of rank 2 is considered in [7.) Below, when dealing with these existence and uniqueness problems, we just state the result and leave details to the reader.

3.4. Irreducible sextics with simple singularities. Our next goal is to relate certain properties of the fundamental group to the kernel $\mathcal{K}_{C}$ of the extension $\tilde{S}_{C} \supset S_{C}$. In this section, we deal with the case of irreducible sextics: it is more transparent and quite sufficient for the purpose of this paper. Reducible sextics are considered in Section 3.5.

3.4.1. Theorem (see [7]). A plane sextic $C$ with simple singularities is irreducible if and only if the group $\mathcal{K}_{C}$ is free of 2-torsion.

3.4.2. Corollary. For an irreducible sextic $C$ with simple singularities one has $\tilde{S}_{C}=\tilde{\Sigma}_{C} \oplus \mathbb{Z} h$ and $\mathcal{K}_{C}=\tilde{\Sigma}_{C} / \Sigma_{C}$.

Proof. One has $\operatorname{discr}(\mathbb{Z} h)=\left\langle\frac{1}{2}\right\rangle$. Hence, the subgroup $\mathcal{K}_{C} \subset \operatorname{discr} \Sigma_{C} \oplus \operatorname{discr}(\mathbb{Z} h)$ belongs entirely to discr $\Sigma_{C}$, and the orthogonal sum decomposition of $S_{C}$ descends to the extension.

3.4.3. Corollary. For an irreducible sextic $C$ with simple singularities one has $\ell_{2}\left(\operatorname{discr} \Sigma_{C}\right)+\mu(C) \leqslant 20$.

Proof. Since $\mathcal{K}_{C}$ is free of 2-torsion, one has $\ell_{2}(\operatorname{discr} \tilde{S})=\ell_{2}(\operatorname{discr} S)=\ell_{2}(\Sigma)+1$. On the other hand, $\ell_{2}(\operatorname{discr} \tilde{S}) \leqslant \operatorname{rk} \tilde{S}^{\perp}=21-\mu(C)$.

3.4.4. Proposition. Let $C$ be an irreducible sextic with simple singularities. Then $\bar{K}_{2}(C)$ splits into eigensubgroups, $\bar{K}_{2}(C)=\operatorname{Ker}\left(\operatorname{tr}_{2}-1\right) \oplus \operatorname{Ker}\left(\operatorname{tr}_{2}+1\right)$, and there are isomorphisms $\operatorname{Ker}\left(\operatorname{tr}_{2}-1\right)=\mathbb{Z} / 3 \mathbb{Z}$ and $\operatorname{Ker}\left(\operatorname{tr}_{2}+1\right)=\operatorname{Ext}\left(\mathcal{K}_{C}, \mathbb{Z}\right)$.

3.4.5. Remark. Proposition 3.4.4, as well as Theorem 3.5.1 below, extend to plane curves of any degree $(4 m+2), m \in \mathbb{Z}$ : one should just replace $\mathbb{Z} / 3 \mathbb{Z}$ with $\mathbb{Z} /(2 m+1) \mathbb{Z}$ everywhere in the statements.

Proof. One has $\bar{K}_{2}(C)=H_{1}(\tilde{X} \backslash(\tilde{C} \cup \tilde{E}))$ and, since $\tilde{X}$ is simply connected, the Poincaré duality establishes an isomorphism

$$
\bar{K}_{2}(C)=\operatorname{Coker}\left[\operatorname{in}^{*}: H^{2}(\tilde{X}) \rightarrow H^{2}(\tilde{C} \cup \tilde{E})\right] .
$$

Let $M=H_{2}(\tilde{C} \cup \tilde{E})$. Since $[\tilde{C}]=3 h \bmod \Sigma$ in $L$, one has $M=\Sigma \oplus(\mathbb{Z} \cdot 3 h)$, the inclusion homomorphism $\mathrm{in}_{*}: M \rightarrow L$ is monic, and, using the universal coefficients formula and replacing $L$ with the primitive hull $\tilde{S}$ of $M$, one concludes that $\bar{K}_{2}(C)=$ $\operatorname{Coker}\left[\tilde{S}^{*} \rightarrow M^{*}\right]=\operatorname{Ext}(\tilde{S} / M, \mathbb{Z})$. Due to Corollary $3.4 .2, \tilde{S} / M=\mathcal{K}_{C} \oplus \mathbb{Z} / 3 \mathbb{Z}$ and, 
in view of Theorem 3.4.1, $\bar{K}_{2}(C)$ is free of 2-torsion. Hence, $\bar{K}_{2}(C)$ splits into eigensubgroups of its order 2 automorphism $\operatorname{tr}_{2}$; obviously, $h$ is $\operatorname{tr}_{2}$-invariant, and the action of $\operatorname{tr}_{2}$ on $\mathcal{K}_{C} \subset \operatorname{discr} \Sigma$ is given by Lemma 3.3.1.

3.4.6. Corollary. Let $C$ be an irreducible sextic with simple singularities. Then there is a canonical one to one correspondence between the set of normal subgroups $N \subset \pi_{1}\left(\mathbb{P}^{2} \backslash C\right)$ with $\pi_{1}\left(\mathbb{P}^{2} \backslash C\right) / N \cong \mathbb{D}_{2 n}, n \geqslant 3$, and the set of subgroups of $\operatorname{Tor}\left(\mathcal{K}_{C}, \mathbb{Z} / n \mathbb{Z}\right)$ isomorphic to $\mathbb{Z} / n \mathbb{Z}$.

Proof. The dihedral quotients $\mathbb{D}_{2 n}$ of the fundamental group are enumerated by the epimorphisms $\operatorname{Ker}\left(\operatorname{tr}_{2}+1\right) \rightarrow \mathbb{Z} / n \mathbb{Z}$ modulo multiplicative units of $(\mathbb{Z} / n \mathbb{Z})$, and the epimorphisms $\operatorname{Ext}\left(\mathcal{K}_{C}, \mathbb{Z}\right) \rightarrow \mathbb{Z} / n \mathbb{Z}$ are the order $n$ elements of the group

$$
\operatorname{Hom}\left(\operatorname{Ext}\left(\mathcal{K}_{C}, \mathbb{Z}\right), \mathbb{Z} / n \mathbb{Z}\right)=\operatorname{Tor}\left(\mathcal{K}_{C}, \mathbb{Z} / n \mathbb{Z}\right) .
$$

(We use the natural isomorphism $\operatorname{Hom}(\operatorname{Ext}(G, \mathbb{Z}), F)=\operatorname{Tor}(G, F)$, which exists for any finite abelian group $G$ and any abelian group $F$.)

3.5. Reducible sextics with simple singularities. For completeness, we prove an analog of Proposition 3.4.4 (and a more precise version of Theorem 3.4.1) for reducible sextics. The results of this section are not used elsewhere in the paper.

3.5.1. Theorem. Let $C$ be a reduced plane sextic with simple singularities, let $C_{1}, \ldots, C_{r}, r \geqslant 2$, be the irreducible components of $C$, and let $c_{i} \in L=L^{*}$ be the class realized by the proper transform $\tilde{C}_{i}$ of $C_{i}$ in $\tilde{X}, 1 \leqslant i \leqslant r$. Then the following statements hold:

(1) each residue $c_{i}$ mod $S$ belongs to the subgroup $\mathcal{K}_{C}^{\prime}=\left\{\alpha \in \mathcal{K}_{C} \mid 2 \alpha=0\right\}$;

(2) the group $\mathcal{K}_{C}^{\prime}$ is generated by the residues $c_{i} \bmod S$, which are subject to the only relation $\sum_{i=1}^{r} c_{i}=0 \bmod S$; in particular, $\ell_{2}\left(\mathcal{K}_{C}\right)=r-1$;

(3) there is an isomorphism Tors $\bar{K}_{2}(C)=(\mathbb{Z} / 3 \mathbb{Z}) \oplus \operatorname{Ext}\left(\mathcal{K}_{C} / \mathcal{K}_{C}^{\prime}, \mathbb{Z}\right)$, so that $\mathrm{tr}_{2}$ acts via +1 and -1 on the first and second summand, respectively;

(4) the group $\bar{K}_{2}(C)$ factors to $(\mathbb{Z} / 3 \mathbb{Z}) \oplus \operatorname{Ext}\left(\mathcal{K}_{C}, \mathbb{Z}\right)$, so that $\operatorname{tr}_{2}$ acts via +1 and -1 on the first and second summand, respectively;

(5) the free part $\bar{K}_{2}(C) /$ Tors $\bar{K}_{2}(C)$ is a free abelian group of rank $r-1$ with the trivial action of $\operatorname{tr}_{2}$.

Proof. As in the proof of Proposition 3.4.4, one has a canonical isomorphism $\bar{K}_{2}(C)=\operatorname{Coker}\left[\tilde{S}^{*} \rightarrow M^{*}\right]$, where $M=H_{2}(\tilde{C} \cup \tilde{E})$. Now, $M$ is a degenerate lattice, its kernel being $\operatorname{Ker}\left[\mathrm{in}_{*}: M \rightarrow L\right] \cong \mathbb{Z}^{r-1}$. (Indeed, modulo $\Sigma$ each class $c_{i}$ is homologous to a multiple of $h$.) This proves statement (5) and gives a natural isomorphism Tors $\bar{K}_{2}(C)=\operatorname{Ext}\left(\tilde{S} / \mathrm{in}_{*} M, \mathbb{Z}\right)$, which reduces (3) to (1) and (2).

To prove statement (4), consider the subgroup $M_{0} \subset M$ spanned by the classes of the exceptional divisors and the total fundamental class $[\tilde{C}]=c_{1}+\ldots+c_{r}$. Since the quotient $M / M_{0}$ is torsion free, in the diagram

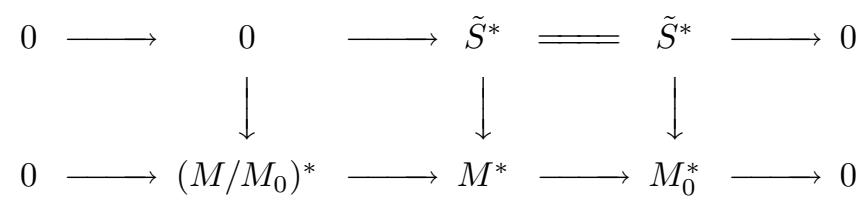

the rows are exact, and the Ker-Coker exact sequence results in an epimorphism $\bar{K}_{2}(C) \rightarrow \operatorname{Ext}\left(\tilde{S} / M_{0}, \mathbb{Z}\right)$. The isomorphism $\tilde{S} / M_{0}=\mathbb{Z} / 3 \mathbb{Z} \oplus \mathcal{K}_{C}$ is established similar to Proposition 3.4.4, the two summands being $S / M_{0}$ and $\operatorname{Ker}\left(\operatorname{tr}_{2}+1\right)$. 
Let $\bar{K}_{2}(C) \rightarrow G$ be the quotient given by (4). The further quotient $G / 2 G$ is an $\mathbb{F}_{2}$-vector space on which $\operatorname{tr}_{2}$ acts identically. Hence, $\pi_{1}\left(\mathbb{P}^{2} \backslash C\right)$ factors to an abelian 2-group $G^{\prime}$ with $\ell_{2}\left(G^{\prime}\right) \geqslant \operatorname{dim}(G / 2 G)=\ell_{2}\left(\mathcal{K}_{C}\right)$. On the other hand, the abelinization of $\pi_{1}\left(\mathbb{P}^{2} \backslash C\right)$ is $\mathbb{Z}^{r-1}$. Thus, $\ell_{2}\left(\mathcal{K}_{C}\right) \leqslant r-1$.

Let $P$ be a simple singular point, and let $\Gamma_{1}, \ldots, \Gamma_{s}$ be the local branches at $P$. The proper pull-back of $\Gamma_{i}$ in $\tilde{X}$ represents a certain class $\gamma_{i} \in \Sigma(P)^{*}, 1 \leqslant i \leqslant s$. These classes can easily be found using the embedded resolution $Y$ described in Section 3.1, it is done in 22]. Below, the result is represented in terms of the basis $\left\{e_{i}^{*}\right\}$ dual to a standard basis $\left\{e_{i}\right\}$ of $\Sigma(P)$. (The representation in terms of the dual basis is very transparent geometrically: one should just list the exceptional divisors that intersect the proper transform of a branch.)

$$
\begin{array}{lll}
\mathbf{A}_{2 k-1}: & \gamma_{1}=\gamma_{2}=e_{k}^{*}, & \mathbf{E}_{6}: \gamma_{1}=e_{3}^{*}, \\
\mathbf{A}_{2 k}: & \gamma_{1}=e_{k}^{*}+e_{k+1}^{*}, & \mathbf{E}_{7}: \gamma_{1}=e_{6}^{*}, \gamma_{2}=e_{7}^{*}, \\
\mathbf{D}_{2 k+1}: & \gamma_{1}=e_{1}^{*}, \gamma_{2}=e_{2 k-1}^{*}, & \mathbf{E}_{8}: \gamma_{1}=e_{8}^{*} . \\
\mathbf{D}_{2 k}: & \gamma_{1}=e_{1}^{*}, \gamma_{2}=e_{2 k-1}^{*}, \gamma_{3}=e_{2 k}^{*}, &
\end{array}
$$

On a case by case basis one can verify that $2 \gamma_{i}=0 \bmod \Sigma(P), i=1, \ldots, s$, and the residues $\gamma_{i} \bmod \Sigma(P)$ generate the subgroup $\{\alpha \in \operatorname{discr} \Sigma(P) \mid 2 \alpha=0\}$ and are subject to the only relation $\sum_{i=1}^{s} \gamma_{i}=0 \bmod \Sigma(P)$.

Now, it is obvious that each class $c_{i}, i=1, \ldots, r$, has the form

$$
c_{i}=\frac{1}{2}\left(\operatorname{deg} C_{i}\right) h+\sum_{\Gamma_{j} \subset C_{i}} \gamma_{j}
$$

the sum running over all singular points of $C$ and all local branches belonging to $C_{i}$. Hence, $2 c_{i}=0 \bmod S$. This proves statement (1) and shows that any nontrivial relation between the residues $c_{i} \bmod S$ has the form $\sum_{i \in I} c_{i}=0 \bmod S$ for some subset $I \subset\{1, \ldots, r\}$. If both $I$ and its complement $\bar{I}$ are not empty, the curves $C^{\prime}=\bigcup_{i \in I} C_{i}$ and $C^{\prime \prime}=\bigcup_{i \in \bar{I}} C_{i}$ intersect in at least one point $P$, which is singular for $C$. Then, not all local branches at $P$ belong to $C^{\prime}$, and from the properties of classes $\gamma_{j}$ stated above it follows that the restriction of $\left[C^{\prime}\right]=\sum_{i \in I} c_{i}$ to $\Sigma(P)^{*}$ is not $0 \bmod \Sigma(P)$.

Since $r$ residues $c_{i} \bmod S \in \mathcal{K}_{C}^{\prime}$ are subject to a single relation, they generate an $\mathbb{F}_{2}$-vector space of dimension $r-1$. On the other hand, as is shown above, $\operatorname{dim} \mathcal{K}_{C}^{\prime}=\ell_{2}\left(\mathcal{K}_{C}\right) \leqslant r-1$. This completes the proof of (2) and, hence, (3).

\section{Curves with simple singularities}

4.1. Curves of torus type: the statements. In this section, we state our principal results concerning sextics of torus type. Proofs are given in Section 4.2.

4.1.1. Theorem. For an irreducible plane sextic $C$ with simple singularities, the following statements are equivalent:

(1) $C$ is of torus type;

(2) the Alexander polynomial $\Delta_{C}(t)$ is nontrivial;

(3) the group $\pi_{1}\left(\mathbb{P}^{2} \backslash C\right)$ factors to the reduced braid group $\mathbb{B}_{3} / \Delta^{2}$;

(4) the group $\pi_{1}\left(\mathbb{P}^{2} \backslash C\right)$ factors to the symmetric group $\mathbb{S}_{3}$. 
A nine cuspidal sextic is an irreducible sextic with nine ordinary cusps, i.e., set of singularities $9 \mathbf{A}_{2}$. These curves are well known; they were used by Zariski to prove the existence of non-special six cuspidal sextics. From the Plücker formulas it follows that any nine cuspidal sextic is dual to a nonsingular cubic curve. In particular, all nine cuspidal sextics are rigidly isotopic.

4.1.2. Theorem. Let $C$ be an irreducible plane sextic with simple singularities, other than a nine cuspidal sextic. Then there are canonical bijections between the following sets:

(1) the set of torus structures on $C$;

(2) the set of normal subgroups $N \subset \pi_{1}\left(\mathbb{P}^{2} \backslash C\right)$ with $\pi_{1}\left(\mathbb{P}^{2} \backslash C\right) / N \cong \mathbb{S}_{3}$;

(3) the projectivization of the $\mathbb{F}_{3}$-vector space $\mathcal{K}_{C} \otimes \mathbb{F}_{3}$.

In the exceptional case of a nine cuspidal sextic, still there is a bijection (2) $\leftrightarrow(3)$ and an injection (1) $\hookrightarrow(3)$; the image of the latter injection misses one point.

The exceptional case in Theorem 4.1 .2 deserves a separate statement.

4.1.3. Theorem. Let $C$ be a nine cuspidal sextic. Then there exists one, and only one, quotient $\pi_{1}\left(\mathbb{P}^{2} \backslash C\right) \rightarrow \mathbb{S}_{3}$ such that the resulting triple plane $p: V \rightarrow \mathbb{P}^{2}$ ramified at $C$ is not a cubic surface. All nine cusps of $C$ are cusps (Whitney pleats) of $p$, and the covering space $V$ is a nonsingular surface of Euler characteristic zero.

Last three theorems give a detailed description of sextics of torus type. In particular, Theorem 4.1.5 lists all sextics admitting more than one torus structure. Recall that the weight $w(C)$ of a sextic $C$ is defined as the total weight of all its singular points, see Definition 3.2.4.

4.1.4. Theorem. Let $C$ be an irreducible sextic with simple singularities. If the weight $w(C)$ is 7 (respectively, 8 or 9$)$, then $\mathcal{K}_{C}=(\mathbb{Z} / 3 \mathbb{Z})^{w(C)-6}$ and $C$ has exactly one (respectively, four or twelve) torus structures. If $w(C)=6$, then $\mathcal{K}_{C}=\mathbb{Z} / 3 \mathbb{Z}$ or $\mathcal{K}_{C}=0$ and $C$ has one or none torus structure, respectively. If $w(C)<6$, then $\ell_{3}\left(\mathcal{K}_{C}\right)=0$ and $C$ is not of torus type.

4.1.5. Theorem. Let $C$ be an irreducible sextic with simple singularities. If $w(C)=9$, then $C$ is a nine cuspidal sextic. If $w(C)=8$, then $C$ has one of the following sets of singularities

$$
\begin{gathered}
8 \mathbf{A}_{2}, \quad 8 \mathbf{A}_{2} \oplus \mathbf{A}_{1}, \quad \mathbf{A}_{5} \oplus 6 \mathbf{A}_{2}, \quad \mathbf{A}_{5} \oplus 6 \mathbf{A}_{2} \oplus \mathbf{A}_{1}, \\
2 \mathbf{A}_{5} \oplus 4 \mathbf{A}_{2}, \quad \mathbf{E}_{6} \oplus 6 \mathbf{A}_{2}, \quad \mathbf{E}_{6} \oplus \mathbf{A}_{5} \oplus 4 \mathbf{A}_{2},
\end{gathered}
$$

each set being realized by at least one rigid isotopy class.

4.1.6. Theorem. Let $C$ be an irreducible sextic with simple singularities and of weight $w(C)=6$, and assume that $C$ has a singular point of weight zero other than a simple node (type $\mathbf{A}_{1}$ ). Then $\mathcal{K}_{C}=\mathbb{Z} / 3 \mathbb{Z}$ and $C$ has exactly one torus structure.

4.1.7. Remark. In the remaining case, $w(C)=6$ and all singular points of weight zero are simple nodes, the same set of singularities may be realized by both sextics of torus type and those not of torus type; they differ by their Alexander polynomials, see abundant vs. non-abundant curves in [4] and [7]. The first example of this kind is due to Zariski [24]. 
4.1.8. Remark. It is quite straightforward to enumerate all sets of singularities realized by sextics of torus type; however, the resulting list is too long and rather meaningless. At present, it is unclear whether each set of singularities is realized by at most one rigid isotopy class of sextics of torus type. In many cases, general theorems of [14] do not apply and, considering the amount of calculations involved, we leave this question open.

4.2. Curves of torus type: the proofs. Given an integer $w>0$, denote by $\mathcal{D}_{w}$ the direct sum of $w$ copies of $\left\langle-\frac{2}{3}\right\rangle$; we regard $\mathcal{D}_{w}$ as an $\mathbb{F}_{3}$-vector space. Let $\alpha_{1}, \ldots, \alpha_{w}$ be some generators of the summands. An isometry of $\mathcal{D}_{w}$ is called admissible if it is the composition of a permutation of $\alpha_{1}, \ldots, \alpha_{w}$ and multiplying some of them by $(-1)$. (One has $\mathcal{D}_{w}=\operatorname{discr}\left(w \mathbf{A}_{2}\right)$, and the admissible isometries are those induced by the isometries of $w \mathbf{A}_{2}$.) Define the weight $w(\delta)$ of an element $\delta \in \mathcal{D}$ as the number of the generators $\alpha_{1}, \ldots, \alpha_{w}$ appearing in $\delta$ with non-zero coefficients. Clearly, $\delta$ is isotropic if and only if $w(\delta)$ is divisible by 3 .

Let $C$ be a reduced (not necessarily irreducible) sextic with simple singularities, and let $w=w(C)$ be the weight of $C$. Consider the subgroup $\mathcal{G}=\mathcal{G}_{C} \subset \operatorname{discr} \Sigma_{C}$ generated by the elements of order 3 . Recall that, for each singular point $P$ of positive weight $w(P)$, the intersection $\mathcal{G} \cap \operatorname{discr} \Sigma(P)$ is generated by a single element $\beta_{P}$ of square $-2 w(P) / 3$. Hence, $\mathcal{G}$ admits an isometric embedding to $\mathcal{D}_{w}$ : split the set $G=\left\{\alpha_{1}, \ldots, \alpha_{w}\right\}$ into disjoint subsets $D_{P}$, assigning $w(P)$ generators to each singular point $P$ of positive weight, and map $\beta_{P}$ to $\sum_{\alpha_{i} \in D_{P}} \alpha_{i}$. Using this embedding, which is defined up to admissible isometry of $\mathcal{D}_{w}$, one can speak about the weights of the elements of $\mathcal{G}$.

4.2.1. Lemma. In the notation above, an extension $\tilde{S}$ of the lattice $S=\Sigma \oplus \mathbb{Z} h$, $h^{2}=2$, defined by an isotropic subgroup $\mathcal{K} \subset \mathcal{G}$ satisfies condition 3.3.2(1) in the definition of configuration if and only if $\mathcal{K}$ has the following property:

(*) each nonzero element of $\mathcal{K}$ has weight at least 6 .

Proof. Given $\gamma \in \mathcal{K}$, the maximal square of a vector $u \in \tilde{S}$ such that $u \bmod S=\gamma$ is $-\frac{2}{3} w(\gamma)$. This maximum equals $(-2)$ if and only if $w(\gamma)=3$.

4.2.2. Lemma. Let $w=9$ (respectively, $w=8$ or $w=6,7$ ), and let $\mathcal{K} \subset \mathcal{D}_{w}$ be an isotropic subspace satisfying condition 4.2.1( $*$ ). Then $\operatorname{dim} \mathcal{K} \leqslant 3$ (respectively, $\operatorname{dim} \mathcal{K} \leqslant 2$ or $\operatorname{dim} \mathcal{K} \leqslant 1$ ). Furthermore, a subspace $\mathcal{K}_{w} \subset \mathcal{D}_{w}$ of maximal dimension is unique up to admissible isometry of $\mathcal{D}_{w}$; it is generated by

$$
\begin{array}{ll}
w=9: & \alpha_{1}+\ldots+\alpha_{9}, \quad \alpha_{1}+\alpha_{2}+\alpha_{3}-\alpha_{4}-\alpha_{5}-\alpha_{6}, \quad \text { and } \\
& \alpha_{1}-\alpha_{2}+\alpha_{4}-\alpha_{5}+\alpha_{7}-\alpha_{8} ; \\
w=8: & \alpha_{1}+\ldots+\alpha_{6} \quad \text { and }-\alpha_{3}-\alpha_{4}+\alpha_{5}+\ldots+\alpha_{8} ; \\
w \leqslant 7: & \alpha_{1}+\ldots+\alpha_{6} .
\end{array}
$$

Proof. All statements can be proved by a case by case analysis. A more conceptual proof for the case $w=8$ is given in 8 , Lemma 5.2. This result implies the dimension estimate for $w \leqslant 7$ (as the subgroup of dimension 2 involves all eight generators of $\mathcal{D}_{8}$ ) and $w=9$. The uniqueness is obvious in the case $w \leqslant 7$; in the case $w=9$ it can be proved geometrically: two non-equivalent isotropic subspaces of $\mathcal{D}_{9}$ of dimension 3 and satisfying 4.2.1(*) would give rise to two distinct configurations extending $9 \mathbf{A}_{2}$ and, in view of Theorem 3.3.4, to two rigid isotopy classes of nine cuspidal sextics. 
4.2.3. Corollary. Let $w \leqslant 9$, and let $\mathcal{K}_{w} \subset \mathcal{D}_{w}$ be the maximal isotropic subspace given by Lemma 4.2.2. If $w \leqslant 8$, then each nonzero element of $\mathcal{K}_{w}$ has weight 6 . If $w=9$, then $\mathcal{K}_{w}$ has two elements of weight 9 and 24 elements of weight 6 .

4.2.4. Lemma. Let $C$ be as above and $w=w(C)$. Assume that the subgroup $\mathcal{G}_{C} \subset \mathcal{D}_{w}$ contains the maximal isotropic subspace $\mathcal{K}_{w}$ given by Lemma 4.2.2, If $w=8$ (respectively, $w=9$ ), then for each singular point $P$ of $C$ one has $w(P) \leqslant 2$ (respectively, $w(P) \leqslant 1$ ).

Proof. According to the definition of the embedding $\mathcal{G}_{C} \hookrightarrow \mathcal{D}_{w}$, the maximal weight of a singular point is bounded by the maximal number $n$ of generators $\alpha_{1}, \ldots, \alpha_{w}$ of $\mathcal{D}_{w}$ appearing in each element $\gamma \in \mathcal{K}_{w}$ with the same coefficient (depending on $\gamma$ ). From the description of $\mathcal{K}_{w}$ given in Lemma 4.2 .2 it follows that $n=1$ for $w=9$ and $n=2$ for $w=8$.

4.2.5. Lemma. In the notation above, there is a natural bijection between the torus structures of $C$ and pairs of opposite elements $\pm \gamma \in \mathcal{K}_{C} \cap \mathcal{G}$ of weight 6 .

Proof. The statement is essentially contained in [7, where the case $w(C)=6$ is considered. Each conic $Q$ as in Theorem 3.2.7 lifts to two disjoint rational curves $\tilde{Q}_{1}, \tilde{Q}_{2}$ in $\tilde{X}$, and a simple calculation using the resolution $Y$ described in Section 3.1 shows that the fundamental classes $\left[\tilde{Q}_{i}\right] \in L$ have the form $\left[\tilde{Q}_{i}\right]=h+\sum_{P \in Q} \bar{\beta}_{P}^{i}$, where, in a standard basis $\left\{e_{i}\right\}$ of $\Sigma(P)$, the elements $\bar{\beta}_{P}^{1,2} \in \Sigma(P)^{*}$ are defined as

$$
\begin{gathered}
\bar{\beta}_{P}^{1}=e_{k}^{*}, \quad \bar{\beta}_{P}^{2}=e_{2 k}^{*} \quad \text { for } P \text { of type } \mathbf{A}_{3 k-1}, \\
\bar{\beta}_{P}^{1}=e_{2}^{*}, \quad \bar{\beta}_{P}^{2}=e_{4}^{*} \quad \text { for } P \text { of type } \mathbf{E}_{6} .
\end{gathered}
$$

One has $\left(\bar{\beta}_{P}^{i}\right)^{2}=-\frac{2}{3} w(P)$, see Lemma 3.2 .5 , and the residues $\bar{\beta}_{P}^{1,2} \bmod \Sigma(P)$ are the two opposite nontrivial order 3 elements of $\operatorname{discr} \Sigma(P)$. Since $2 \sum_{P \in Q} w(P)=$ $C \cdot Q=12$, the residues $\left(\left[Q_{i}\right]-h\right) \bmod \Sigma$ form a pair of opposite elements of $\mathcal{K}_{C}$ of weight 6 .

Conversely, any order 3 element $\gamma \in \mathcal{K}_{C}$ can be represented (possibly, after reordering $\bar{\beta}^{1}$ 's and $\bar{\beta}^{2}$ 's) as the residue of the class $\bar{\gamma}=\sum_{P \in J} \bar{\beta}_{P}^{1}$, the sum running over a subset $J$ of the set of singular points with $\sum_{P \in J} w(P)=w(\gamma)$. If $w(\gamma)=6$, one has $(\bar{\gamma}+h)^{2}=-2$ and, since obviously $\bar{\gamma}+h \in \operatorname{Pic} \tilde{X}$, the Riemann-Roch theorem implies that $\bar{\gamma}+h$ is realized by a (possibly reducible) rational curve $\tilde{Q}$ in $\tilde{X}$. The image of $\tilde{Q}$ in $\mathbb{P}^{2}$ is a conic $Q$ as in Theorem 3.2.7.

4.2.6. Remark. In the proof of Lemma 4.2 .5 , the lifts $\tilde{Q}_{1}, \tilde{Q}_{2}$ are the connected components of the proper pull-back of $Q$ provided that $Q$ is nonsingular at each singular point of $C$. If $Q$ is singular at a point $P$ of $C$, then $P$ is of type $\mathbf{A}_{3 k-1}$, $k \geqslant 2$, and a proper pull-back realizes (locally) a class of the form $e_{1}^{*}+e_{k-1}^{*}$. In this case, one should include into $\tilde{Q}_{1}$ several exceptional divisors, according to the relation $e_{1}^{*}+e_{k-1}^{*}+e_{1}+\ldots+e_{k-1}=e_{k}^{*}$. We leave details to the reader.

4.2.7. Lemma. Let $C$ be an irreducible sextic with simple singularities, and let $w(C) \geqslant 7$. Then $\mathcal{K}_{C}$ has elements of order 3 .

Proof. According to 4], the Alexander polynomial $\Delta_{C}(t)$ is $\left(t^{2}-t+1\right)^{s}$, where $s$ is the superabundance of the linear system $\mathcal{L}_{5}$ of conics satisfying certain explicitly described conditions at the singular points of $C$. In particular, each singular point $P$ of positive weight $w(P)$ imposes $d_{5 / 6}(P)=w(P)$ conditions, see Remark 3.2.6. 
Hence, the virtual dimension of $\mathcal{L}_{5}$ is less than -1 and $\Delta_{C}(t) \neq 1$. The statement of the lemma follows from Proposition 3.1.1 and Corollary 3.4.6.

Proof of Theorems 4.1.4 and 4.1.5. First, note that the group $\mathcal{K}_{C}$ has no elements of order 9. Indeed, order 9 elements are only present in discr $\mathbf{A}_{8}, 2$ discr $\mathbf{A}_{8}$, or discr $\mathbf{A}_{17}$. However, none of these discriminants contains an order 9 element whose square is $0 \bmod \frac{1}{3} \mathbb{Z}$ (so that it could be compensated by the square of an order 3 element coming from other singular points).

Fix an irreducible sextic $C$ with simple singularities and introduce the following notation:

- $w=w(C)=$ the weight of $C$;

- $m=$ the total number of the singular points $P$ of $C$ with $w(P)>0$;

$-e=$ the number of singular points of type $\mathbf{E}_{6}$;

- $\mu^{\prime}=$ the total Milnor number of the singular points of $C$ of weight zero;

$-\kappa=\operatorname{dim} \mathcal{K}_{C} \otimes \mathbb{F}_{3}$.

The total Milnor number of the singularities of $C$ is $\mu=3 w-m+e+\mu^{\prime}$; since $m \leqslant w$, the inequality $\mu \leqslant 19$ implies that $w \leqslant 9$.

One has $\ell_{3}(\operatorname{discr} \Sigma)=m$. Hence, $m-2 \kappa \leqslant \ell_{3}(\operatorname{discr} \tilde{S}) \leqslant \operatorname{rk} \tilde{S}^{\perp}=21-\mu$, i.e., $2 \kappa \geqslant 3 w+e+\mu^{\prime}-21$. This inequality, combined with Lemma 4.2.2, yields:

- if $w=9$, then $\kappa=3$ and $e=\mu^{\prime}=0$;

- if $w=8$, then $\kappa=2$ and $e+\mu^{\prime} \leqslant 1$;

- if $w=7$, then $\kappa=1$ (due to Lemma 4.2.7) and $e+\mu^{\prime} \leqslant 2$;

- if $w=6$, then $\kappa \leqslant 1$ and $e+\mu^{\prime} \leqslant 2 \kappa+3$.

In all cases with $w \geqslant 6$ one has $\mu^{\prime} \leqslant 5$. Furthermore, whenever $w \geqslant 7$, the subgroup $\mathcal{K}_{C} \subset \mathcal{G}_{C} \subset \mathcal{D}_{w}$ is the maximal subspace $\mathcal{K}_{w}$ given by Lemma 4.2.2.

To complete the proof of Theorem 4.1.4, it remains to show that, whenever $w \geqslant 6$ and $p \neq 3$ is a prime, the group $\mathcal{K}_{C}$ is free of $p$-torsion. Since $C$ is irreducible, $\mathcal{K}_{C}$ is free of 2-torsion, see Theorem 3.4.1. If $p \geqslant 5, p$-torsion elements are only present in the discriminants discr $\mathbf{A}_{i}$ with $p \mid(i+1)$. If $w\left(\mathbf{A}_{i}\right)=0$, then $i \leqslant \mu^{\prime} \leqslant 5$ and the only possibility is $p=5, i=4$. If $w\left(\mathbf{A}_{i}\right)>0$, then $3 p \mid(i+1)$ and, since $i \leqslant 19$, the only possibility is $p=5, i=14$. In this case $m \leqslant w-4$, and the inequality $\mu \leqslant 19$ implies that $e+\mu^{\prime} \leqslant 3$, i.e., there are no other points with order 5 elements in the discriminant. Thus, one has $p=5$ and the 5 -torsion of discr $\Sigma$ comes either from a single point of type $\mathbf{A}_{4}$ or from a single point of type $\mathbf{A}_{14}$. However, neither discr $\mathbf{A}_{4}$ nor discr $\mathbf{A}_{14}$ have an isotropic element of order 5.

Prove Theorem 4.1.5. If $w(C)=9$, the statement follows immediately from Lemma 4.2.4 and the fact that $\mu^{\prime}=0$. If $w(C)=8$, the possible sets of singularities are easily enumerated using the inequality $e+\mu^{\prime} \leqslant 1$ above and Lemma 4.2.4, which only allows $\mathbf{A}_{1}, \mathbf{A}_{2}$, or $\mathbf{E}_{6}$ for a singularity of positive weight. The sets of singularities $2 \mathbf{A}_{5} \oplus 4 \mathbf{A}_{2} \oplus \mathbf{A}_{1}$ and $3 \mathbf{A}_{5} \oplus 2 \mathbf{A}_{2}$ are ruled out by Corollary 3.4 .3 , the realizability of the seven sets listed in the theorem follows from Theorem 3.3 .4 and Theorem 1.10.1 in [14, see Remark 3.3.5.

Proof of Theorem 4.1.6. Similar to Lemma 4.2.7, we use the results of 4 (see Remark 3.2.6) to evaluate the Alexander polynomial $\Delta_{C}(t)$. For each singular point $P$ other than $\mathbf{A}_{1}$, one has $d_{5 / 6}(P) \geqslant 1$. Hence, the total number of conditions on the conics in $\mathcal{L}_{5}$ is $\sum d_{5 / 6}(P) \geqslant w(C)+1=7$. Then, the virtual dimension of $\mathcal{L}_{5}$ is less than -1 , one has $\Delta_{C}(t) \neq 1$, from Proposition 3.1.1 and Corollary 3.4.6 it follows that $\mathcal{K}_{C}$ has 3 -torsion, and Theorem 4.1 .4 applies. 
Proof of Theorem 4.1.1. The implication (3) $\Longrightarrow(4)$ is obvious, $(2) \Longrightarrow(4)$ is given by Proposition 3.1.1, and (1) $\Longrightarrow(3)$ and $(1) \Longrightarrow(2)$ are given by Proposition 3.2 .3 . Thus, it remains to show that (4) implies (1).

Let $C$ satisfy condition (4). Due to Corollary 3.4.6, the group $\mathcal{K}_{C}$ has elements of order 3 and, comparing Theorem 4.1.4 and Lemma 4.2.2, one concludes that $\mathcal{K}_{C} \subset \mathcal{G}_{C} \subset \mathcal{D}_{w(C)}$ is the maximal isotropic subspace given by Lemma 4.2.2. Then, Corollary 4.2 .3 and Lemma 4.2 .5 imply that $C$ is of torus type.

Proof of Theorem 4.1.2. The bijection $(2) \leftrightarrow(3)$ is given by Corollary 3.4.6: since $\mathcal{K}_{C}$ has no elements of order 9 , the order 3 subgroups in $\operatorname{Tor}\left(\mathcal{K}_{C}, \mathbb{F}_{3}\right)$ are in a one to one correspondence with those in $\mathcal{K}_{C} \otimes \mathbb{F}_{3}$.

The bijection (1) $\leftrightarrow(3)$ is that given by Lemma 4.2.5 in view of Theorems 4.1.4 and 4.1.5, the only exception is the pair of opposite elements of weight 9 that exist in the case of a nine cuspidal sextic.

Proof of Theorem 4.1.3. The triple plane described in the statement corresponds to the two elements $\pm \gamma \in \mathcal{K}_{C}$ of weight 9 . In general, the cusps of the triple plane arising from an element $\gamma \in \mathcal{K}_{C}$ can be detected as the singular points $P$ of $C$ with the following property:

$(*)$ the composition $\pi_{1}\left(U_{P} \backslash C\right) \rightarrow \pi_{1}\left(\mathbb{P}^{2} \backslash C\right) \rightarrow \mathbb{S}_{6}$ is an epimorphism, where $U_{P} \subset \mathbb{P}^{2}$ is a Milnor ball about $P$.

Let $\tilde{U}$ be the minimal resolution of the double covering of $U_{P}$ ramified at $C$. Then, as in Proposition 3.4.4, the abelinization of the kernel of the corresponding homomorphism $\pi_{1}\left(U_{P} \backslash C\right) \rightarrow \mathbb{Z} / 2 \mathbb{Z}$ is given by $H_{1}(\partial \tilde{U})=\operatorname{discr} H_{2}(\tilde{U})=\operatorname{discr} \Sigma(P)$. Hence, a point $P$ has property $(*)$ if and only if the restriction of $\gamma$ to $\operatorname{discr} \Sigma(P)=$ $\Sigma(P)^{*} / \Sigma(P)$ is nonzero; it holds for all nine cusps if $w(\gamma)=9$.

The Euler characteristic of $V$ is found from the Riemann-Hurwitz formula.

4.3. Other curves admitting dihedral coverings. An irreducible sextic is called special if its fundamental group factors to a dihedral group $\mathbb{D}_{2 n}, n \geqslant 3$. Theorem 4.1.1 implies that all irreducible sextics of torus type are special. In this section, we enumerate other special sextics with simple singularities.

4.3.1. Theorem. Let $C$ be an irreducible plane sextic with simple singularities. Then the group $\operatorname{Ker}\left(\operatorname{tr}_{2}+1\right)$ is either $(\mathbb{Z} / 3 \mathbb{Z})^{m}, 0 \leqslant m \leqslant 3$, or $\mathbb{Z} / 5 \mathbb{Z}$, or $\mathbb{Z} / 7 \mathbb{Z}$.

4.3.2. Corollary. Let $C$ be an irreducible plane sextic with simple singularities. Then any dihedral quotient of $\pi_{1}\left(\mathbb{P}^{2} \backslash C\right)$ is either $\mathbb{D}_{6} \cong \mathbb{S}_{3}$ or $\mathbb{D}_{10}$ or $\mathbb{D}_{14}$.

4.3.3. Theorem. There are two rigid isotopy classes of special sextics with simple singularities whose fundamental group factors to $\mathbb{D}_{14}$; their sets of singularities are $3 \mathbf{A}_{6}$ and $3 \mathbf{A}_{6} \oplus \mathbf{A}_{1}$. The set of singularities $3 \mathbf{A}_{6}$ can also be realized by a non-special irreducible sextic.

The two special sextics above can be characterized as follows: there is an ordering $P_{1}, P_{2}, P_{3}$ of the three $\mathbf{A}_{6}$ points such that, for every cyclic permutation $\left(i_{1} i_{2} i_{3}\right)$, there is a conic whose local intersection index with $C$ at $P_{i_{k}}$ equals $2 k$.

4.3.4. Theorem. There are eight rigid isotopy classes of special sextics with simple singularities whose fundamental group factors to $\mathbb{D}_{10}$; each class is determined by its set of singularities, which is one of the following:

$4 \mathbf{A}_{4}, \quad 4 \mathbf{A}_{4} \oplus \mathbf{A}_{1}, \quad 4 \mathbf{A}_{4} \oplus 2 \mathbf{A}_{1}, \quad 4 \mathbf{A}_{4} \oplus \mathbf{A}_{2}$,

$\mathbf{A}_{9} \oplus 2 \mathbf{A}_{4}, \quad \mathbf{A}_{9} \oplus 2 \mathbf{A}_{4} \oplus \mathbf{A}_{1}, \quad \mathbf{A}_{9} \oplus 2 \mathbf{A}_{4} \oplus \mathbf{A}_{2}, \quad 2 \mathbf{A}_{9}$. 
The sets of singularities $4 \mathbf{A}_{4}, 4 \mathbf{A}_{4} \oplus \mathbf{A}_{1}, \mathbf{A}_{9} \oplus 2 \mathbf{A}_{4}, \mathbf{A}_{9} \oplus 2 \mathbf{A}_{4} \oplus \mathbf{A}_{1}$, and $2 \mathbf{A}_{9}$ are also realized by non-special irreducible sextics.

The eight special sextics above can be characterized as follows: there are two conics $Q_{1}, Q_{2}$ with the following properties:

- $Q_{1}$ and $Q_{2}$ intersect transversally at each singular point of $C$ of type $\mathbf{A}_{4}$, and they have a simple tangency at each singular point of $C$ of type $\mathbf{A}_{9}$;

- at each singular point of type $\mathbf{A}_{4}$, the local intersection indices of $C$ with the two conics are 2 and 4 ;

- at each singular point of type $\mathbf{A}_{9}$, the local intersection indices of $C$ with the two conics are 4 and 8.

4.3.5. Remark. The Alexander polynomials of all curves listed in Theorems 4.3 .3 and 4.3 .4 are trivial, e.g., due to Proposition 3.1.1. Hence, the sets of singularities $3 \mathbf{A}_{6}, 4 \mathbf{A}_{4}, 4 \mathbf{A}_{4} \oplus \mathbf{A}_{1}, \mathbf{A}_{9} \oplus 2 \mathbf{A}_{4}, \mathbf{A}_{9} \oplus 2 \mathbf{A}_{4} \oplus \mathbf{A}_{1}$, and $2 \mathbf{A}_{9}$ that are realized by both special and non-special curves give rise to Alexander equivalent Zariski pairs of irreducible sextics. This means that two irreducible curves $C_{1}, C_{2}$ share the same set of singularities and Alexander polynomial but have non-diffeomorphic complements $\mathbb{P}^{2} \backslash C_{i}$ (see [2] for precise definitions). In our case, the fundamental groups $\pi_{1}\left(\mathbb{P}^{2} \backslash C\right)$ differ: one does and the other does not admit dihedral quotients.

Proof of Theorem 4.3.1. Since $C$ is irreducible, $\mathcal{K}_{C}$ is free of 2-torsion. The case when $\mathcal{K}_{C}$ has 3 -torsion is considered in Theorem 4.1.4. For a prime $p \geqslant 5$, any simple singularity whose discriminant has elements of order $p^{a}$ is of type $\mathbf{A}_{i}$ with $p^{a} \mid(i+1)$. Since the total Milnor number $\mu \leqslant 19$, one has $p^{a}=5,7,11,13,17$, or 19. In the last four cases, $p=11,13,17$, or 19 , the set of singularities has at most one point with $p$-torsion in the discriminant, which is of type $\mathbf{A}_{p-1}$; however, $\operatorname{discr} \mathbf{A}_{p-1}=\left\langle-\frac{p-1}{p}\right\rangle$ does not have isotropic elements of order $p$. The remaining cases $p=7$ and $p=5$ are considered in Theorems 4.3 .3 and 4.3.4, respectively. In particular, it is shown that the $p$-primary part of $\mathcal{K}_{C}$ is $\mathbb{Z} / p \mathbb{Z}$. Comparing the sets of singularities listed in Theorems 4.3.3 and 4.3.4, one immediately concludes that $\mathcal{K}_{C}$ cannot have both 7 - and 5-torsion.

Proof of Theorem 4.3.3. Since $\mu \leqslant 19$, the part of $\Sigma$ whose discriminant has 7torsion is either $a \mathbf{A}_{6}, 1 \leqslant a \leqslant 3$, or $\mathbf{A}_{13} \oplus \mathbf{A}_{6}$; it is easy to see that only $\operatorname{discr}\left(3 \mathbf{A}_{6}\right)$ contains an order 7 isotropic element, and it is unique up to isometry of $\Sigma$. Besides, since $\operatorname{discr}\left(3 \mathbf{A}_{6}\right)=(\mathbb{Z} / 7 \mathbb{Z})^{3}$ and the form is nondegenerate, this group cannot contain an isotropic subgroup larger than $\mathbb{Z} / 7 \mathbb{Z}$. These observations restrict the possible sets of singularities to those listed in the statement. The existence of all three curves mentioned in the statement and the uniqueness of the two special curves are straightforward, see Theorem 3.3.4 and Remark 3.3.5; the set of singularities $3 \mathbf{A}_{6} \oplus \mathbf{A}_{1}$ cannot be realized by a non-special curve since for such a curve one would have $\ell_{7}(\operatorname{discr} \tilde{S})=3>2=\operatorname{rk} \tilde{S}^{\perp}$.

The characterization of the special curves in terms of conics is obtained similar to Lemma 4.2.5. Let $P_{i}, i=1,2,3$, be the three points of type $\mathbf{A}_{6}$, and denote by $e_{i j}, j=1, \ldots, 6$, a standard basis of $\Sigma\left(P_{i}\right)$. Then, up to a symmetry of the Dynkin graph, an isotropic element in discr $\Sigma$ is given by $\gamma=e_{11}^{*}+e_{22}^{*}+e_{33}^{*} \bmod \Sigma$. The class $e_{11}^{*}+e_{22}^{*}+e_{33}^{*}+h$ has square $(-2)$; hence, it is realized by a rational curve in $\tilde{X}$, which projects to a conic in $\mathbb{P}^{2}$. The two other conics are obtained from the classes $2 \gamma=e_{12}^{*}+e_{24}^{*}+e_{36}^{*} \bmod \Sigma$ and $3 \gamma=e_{13}^{*}+e_{26}^{*}+e_{32}^{*} \bmod \Sigma$. Conversely, 
each conic as in the statement lifts to a rational curve in $\tilde{X}$ that realizes an order 7 element in discr $\Sigma$.

Proof of Theorem 4.3.4. The singularities whose discriminants contain elements of order 5 are $\mathbf{A}_{4}, \mathbf{A}_{9}, \mathbf{A}_{14}$, and $\mathbf{A}_{19}$. The only imprimitive finite index extension of $2 \mathbf{A}_{4}$ is $2 \mathbf{A}_{4} \subset \mathbf{E}_{8}$; it violates condition $3.3 .2(1)$ in the definition of configuration. With this possibility ruled out, the discriminants containing an order 5 isotropic subgroup are those of $4 \mathbf{A}_{4}, \mathbf{A}_{9} \oplus 2 \mathbf{A}_{4}$, and $2 \mathbf{A}_{9}$. In each case, the subgroup is unique up to a symmetry of the Dynkin graph; it is generated by the residue $\gamma=\bar{\gamma} \bmod \Sigma$, where $\bar{\gamma}$ is given by

$$
e_{11}^{*}+e_{21}^{*}+e_{32}^{*}+e_{42}^{*}, \quad f_{14}^{*}+e_{11}^{*}+e_{21}^{*}, \quad \text { and } \quad f_{14}^{*}+f_{22}^{*},
$$

respectively. Here, $\left\{e_{i j}\right\}, j=1, \ldots, 4$, is a standard basis in the $i$-th copy of $\mathbf{A}_{4}$, and $\left\{f_{k j}\right\}, j=1, \ldots, 9$, is a standard basis in the $k$-th copy of $\mathbf{A}_{9}$. Similar to Lemma 4.2.5, these expressions give a characterization of the special curves in terms of conics: the class $\bar{\gamma}+h$ has square $(-2)$ and is realized by a rational curve in $\tilde{X}$; its projection to $\mathbb{P}^{2}$ is one of the two conics. The other conic is obtained from a similar representation of $2 \gamma$.

The rest of the theorem is an application of Theorem 3.3.4 and Nikulin's results on lattices. The sets of singularities $4 \mathbf{A}_{4} \oplus 3 \mathbf{A}_{1}, \mathbf{A}_{9} \oplus 2 \mathbf{A}_{4} \oplus 2 \mathbf{A}_{1}$, and $2 \mathbf{A}_{9} \oplus \mathbf{A}_{1}$ cannot be realized by irreducible curves due to the genus formula (alternatively, due to Corollary 3.4.3). The sets of singularities $4 \mathbf{A}_{4} \oplus \mathbf{A}_{2} \oplus \mathbf{A}_{1}$ and $4 \mathbf{A}_{4} \oplus \mathbf{A}_{3}$ do not extend to abstract homological types due to Theorem 1.10.1 in [14, see Remark 3.3.5. The (non-)existence of the other curves mentioned in the statement is given by Theorem 3.3.4, see Remark 3.3.5. The uniqueness of the special curves is also given by Theorem 3.3.4 in most cases one can apply either Theorem 1.14.2 in 14] or Theorem 1.13.2 in [14] and the fact that all automorphisms of discr $S$ are realized by isometries of $\Sigma$. (The last statement is true in all cases except $\mathbf{A}_{9} \oplus 2 \mathbf{A}_{4} \oplus \mathbf{A}_{1}$.) We leave details to the reader.

\section{Some CURVES WITH A NON-SIMPLE SINGULAR POINT}

In this concluding section we try to substantiate Conjecture 1.1.1(1) extended to all irreducible sextics. Here, we consider sextics with a non-simple singular point adjacent to $\mathbf{X}_{9}$, i.e., a point of multiplicity 4 or 5 . The only remaining case of a singular point adjacent to $\mathbf{J}_{10}$ will be dealt with in a separate paper.

5.1. Sextics with a singular point of multiplicity 5 . This case is trivial: due to [5], any irreducible sextic with a singular point of multiplicity 5 has abelian fundamental group and, hence, trivial Alexander polynomial. Note that Proposition 3.2 .3 implies that none of such sextics is of torus type.

5.2. Sextics with a singular point of multiplicity 4 . The rigid isotopy classification of plane curves $C$ with a singular point $P$ of multiplicity $\operatorname{deg} C-2$ is found in 3]. Let $m=\operatorname{deg} C$. In appropriate coordinates $\left(x_{0}: x_{1}: x_{2}\right)$ the curve is given by a polynomial of the form

$$
x_{0}^{2} a\left(x_{1}, x_{2}\right)+x_{0} b\left(x_{1}, x_{2}\right)+c\left(x_{1}, x_{2}\right),
$$

where $a, b$, and $c$ are some homogeneous polynomials of degree $m-2, m-1$, and $m$, respectively. The discriminant $D=b^{2}-4 a c$ has degree $2 m-2$. (It is required that 
$D$ is not identically zero.) Since $C$ is assumed irreducible, $a, b$, and $c$ should not have common roots. Let $x_{i}, i=1, \ldots, k$, be all distinct roots of $a D$. The formula of $C$ is defined as the (unordered) set $\left\{\left(p_{i}, q_{i}\right)\right\}, i=1, \ldots, k$, where $p_{i}$ and $q_{i}$ are the multiplicities of $x_{i}$ in $a$ and $D$, respectively. The formula of an irreducible curve of degree $m$ has the following properties:

(1) $\sum_{i=1}^{k} p_{i}=m-2$, and $\sum_{i=1}^{k} q_{i}=2 m-2$;

(2) for each $i$, either $p_{i}=q_{i}$ or the smallest of $p_{i}, q_{i}$ is even;

(3) at least one of $q_{i}$ is odd.

An elementary equivalence of a formula is replacing two pairs $(1,0),(0,1)$ with one pair $(1,1)$. Geometrically, this procedure means that a 'vertical' tangency point of $C$ disappears at infinity making $P$ an inflection point of one of its smooth branches. Clearly, this is a rigid isotopy.

5.2.1. Theorem (see [3]). Two irreducible curves of degree $m$, each with a singular point of multiplicity $m-2$, are rigidly isotopic if and only if their formulas are related by a sequence of elementary equivalences and their inverses. Any set of pairs of nonnegative integers satisfying conditions (1) $-(\sqrt{3})$ above is realized as the formula of an irreducible curve of degree $m$.

Let $G_{p}, p=2,4$, be the group given by

$$
G_{p}=\left\langle u, v \mid u^{p}=v^{p},(u v)^{2} u=v(u v)^{2}, v^{4}=(u v)^{5}\right\rangle .
$$

In [5], it is shown that $\left|G_{4}\right|=\infty$ and $\left|G_{2}\right|=30$; there is a split exact sequence

$$
1 \rightarrow \mathbb{F}_{5}[t] /(t+1) \rightarrow G_{2} \rightarrow \mathbb{Z} / 6 \mathbb{Z} \rightarrow 1
$$

$t$ being the conjugation action on the kernel of a generator of $\mathbb{Z} / 6 \mathbb{Z}$. The Alexander polynomials of both $G_{2}$ and $G_{4}$ are trivial.

5.2.2. Theorem. Irreducible sextics $C$ with a singular point of multiplicity 4 and nonabelian fundamental group form seven rigid isotopy classes, one class for each of the following formulas:

- $\{(2,0),(2,0),(0,5),(0,5)\}$, with $\pi_{1}\left(\mathbb{P}^{2} \backslash C\right)=G_{2} \cong \mathbb{D}_{6} \times(\mathbb{Z} / 3 \mathbb{Z}) ;$

- $\{(4,0),(0,5),(0,5)\}$, with $\pi_{1}\left(\mathbb{P}^{2} \backslash C\right)=G_{4}$;

- $\{(2,2),(2,2),(0,3),(0,3)\}$;

- $\{(2,5),(2,2),(0,3)\}$;

$-\quad\{(2,5),(2,5)\}$;

- $\{(4,4),(0,3),(0,3)\}$;

- $\{(4,7),(0,3)\}$.

In the first two cases, the curve is not of torus type and one has $\Delta_{C}(t)=1$; in the last five cases, the curve is of torus type and one has $\pi_{1}\left(\mathbb{P}^{2} \backslash C\right)=\mathbb{B}_{3} / \Delta^{2}$.

Proof. The fundamental group of a curve $C$ of degree $m$ with a singular point of multiplicity $m-2$ is described in $[5$. If $m=6$, it is easy to enumerate all formulas satisfying conditions (1) $-(3)$ above and select those that give rise to non-abelian fundamental groups. Then, Theorem 5.2 .1 would apply to give one rigid isotopy class for each formula found.

In fact, for $\pi_{1}\left(\mathbb{P}^{2} \backslash C\right)$ not to be abelian, one must have $p_{i} \neq 1$ for all $i$; since also $\sum p_{i}=4$, the nonzero entries $p_{i}$ are either 2 and 2 or 4 . Another necessary 
condition is that $q=$ g.c.d. $\left\{q_{i}-p_{i}\right\}_{q_{i}>p_{i}}$ must be larger than 1 and, in view of (3) above, $q$ must also be odd. These restrictions leave the seven formulas listed in the statement.

For the last five formulas one has $q_{i} \geqslant p_{i}$ and $3 \mid\left(q_{i}-p_{i}\right)$ for each $i$. Hence, $a$ is a square, $a \mid D$, and $D / a$ is a cube. Let $a=p^{2}$ and $D=-4 p^{2} s^{3}$. Then $p \mid b, b=2 p q$, and $c=q^{2}+s^{3}$. Thus, the equation of $C$ has the form

$$
\left(x_{0} p+q\right)^{2}+s^{3}=0,
$$

i.e., $C$ is of torus type.

\section{REFERENCES}

1. V. I. Arnol'd, A. N. Varchenko, S. M. Guser̆n-Zade, Singularities of differentiable maps, vol. I. The classification of critical points, caustics and wave fronts, Nauka, Moscow, 1982; vol. II. Monodromy and asymptotics of integrals, Nauka, Moscow, 1984 (Russian); English translation: Monographs in Mathematics, vol. 82, Birkhäuser Boston, Inc., Boston, MA, 1985; vol. 83, Birkhäuser Boston, Inc., Boston, MA, 1988.

2. E. Artal, J. Carmona, Zariski pairs, fundamental groups, and Alexander polynomials, J. Math. Soc. Japan 50 (1998), 521-543.

3. A. Degtyarev, Isotopy classification of complex plane projective curves of degree 5, Algebra i Analis 1 (1989), no. 4, 78-101 (Russian); English transl. in Leningrad Math. J. 1 (1990), no. $4,881-904$.

4. A. Degtyarev, Alexander polynomial of a curve of degree six, J. Knot Theory Ramif. 3 (1994), 439-454.

5. A. Degtyarev, Quintics in $\mathbb{C p}^{2}$ with nonabelian fundamental group, Algebra i Analis 11 (1999), no. 5, 130-151 (Russian); English transl. in Leningrad Math. J. 11 (2000), no. 5, 809-826.

6. A. Degtyarev, A divisibility theorem for the Alexander polynomial of a plane algebraic curve, Zap. Nauchn. Sem. S.-Peterburg. Otdel. Mat. Inst. Steklov. (POMI), Geom. i Topol. 7, vol. 280, 2001, pp. 146-156, 300 (Russian); English transl. in J. Math. Sci. (N.Y.) 119 (2004), no. $2,205-210$.

7. A. Degtyarev, On deformations of singular plane sextics (to appear).

8. A. Degtyarev, T. Ekedahl, I. Itenberg, B. Shapiro, M. Shapiro, On total reality of meromorphic functions (to appear).

9. A. H. Durfee, Fifteen characterizations of rational double points and simple critical points, Enseign. Math. (2) 25 (1979), no. 1-2, 131-163.

10. C. Eyral, M. Oka, On the fundamental groups of the complements of plane singular sextics, J. Math. Soc. Japan 57 (2005), no. 1, 37-54.

11. A. Libgober, Alexander polynomial of plane algebraic curves and cyclic multiple planes, Duke Math. J. 49 (1982), no. 4, 833-851.

12. A. Libgober, Alexander modules of plane algebraic curves, Contemporary Math. 20 (1983), 231-247.

13. B. G. Moishezon, Stable branch curves and braid monodromies, Lect. Notes Math. 862 (1981), 107-192.

14. V. V. Nikulin, Integer quadratic forms and some of their geometrical applications, Izv. Akad. Nauk SSSR, Ser. Mat 43 (1979), 111-177 (Russian); English transl. in Math. USSR-Izv. 43 (1980), 103-167.

15. M. V. Nori, Zariski conjecture and related problems, Ann. Sci. Éc. Norm. Sup., 4 série 16 (1983), 305-344.

16. M. Oka, Some plane curves whose complement have non-abelian fundamental group, Math. Ann. 218 (1978), 55-65.

17. M. Oka, Alexander polynomial of sextics, J. Knot Theory Ramif. 12 (2003), no. 5, 619-636.

18. M. Oka, A survey on Alexander polynomials of plane curves, Séminair \& Congrès 10 (2005), 209-232.

19. H. Tokunaga, (2,3)-torus sextics and the Albanese images of 6-fold cyclic multiple planes, Kodai Math. J. 22 (1999), no. 2, 222-242. 
20. H. Tokunaga, Introduction to branched Galois covers, Vietnam J. Math. 33 (2005), Special Issue $97-107$.

21. H. Tokunaga, A note on triple covers of $\mathbb{P}^{2}$ (to appear).

22. J.-G. Yang, Sextic curves with simple singularities, Tohoku Math. J. (2) 48 (1996), no. 2, 203-227.

23. O. Zariski, On the problem of existence of algebraic functions of two variables possessing a given branch curve, Amer. J. Math. 51 (1929), 305-328.

24. O. Zariski, On the irregularity of cyclic multiple planes, Ann. Math. 32 (1931), 485-511.

Department of Mathematics, Bilkent University, 06800 Ankara, Turkey

E-mail address: degt@fen.bilkent.edu.tr 